\title{
1 Auf der Suche nach den Landjuden
}

\section{Konventionen der Judendarstellung: Johann Martin Miller und der Typus des »armen Juden«}

\author{
Als Siegwart, der empfindsame Held des gleichnamigen Erfolgsromans ${ }^{64}$ von \\ Johann Martin Miller (1776), zu einer Fahrt von Günzburg nach Ingolstadt auf- \\ bricht, befindet er sich in Gesellschaft. Neben dem »Kondukteur« nehmen ein \\ junger bayerischer Offizier und »ein Jude« in der Postkutsche Platz. Darauf ereig- \\ net sich folgende Szene:
}

Der Offizier, und der Kondukteur fiengen an, den armen Juden auf alle Art zu necken. Keine halbe Stunde durfte er auf seiner Stelle sitzen bleiben. Bald fiels dem Offizier ein, vorwärts, bald wieder rückwärts zu fahren. Der Jude ließ sich alles gefallen, und setzte sich stillschweigend hin, wohin mans wollte. Endlich fiel dem Kondukteur ein, daß er ein wildes Schwein auf dem Wagen habe. Er sagte dem Juden, er soll sich weiter hinten hin im Postwagen setzen. Der Jude thats. Hierauf fieng der Kondukteur mit dem Offizier ein lautes Gelächter an. Mauschel, Mauschel, hast du Gelüst zu Schweinefleisch? Seht mir doch, da setzt er sich neben die Bache hin! Indem zog der Kondukteur die Decke weg, unter der das

64 Millers Siegwart hatte ebenso wie Goethes Werther eine »ungeheure Wirkung auf die Zeitgenossen, die greifbar wird in der großen Zahl der Nachdrucke, der Nachahmungen und der Parodien auf ihn « (Bernd Breitenbruch: Johann Martin Miller. 1750-1814. Liederdichter des Göttinger Hain. Romancier. Prediger am Ulmer Münster. Ausstellung zum 250. Geburtstag. Weißenhorn: Konrad 2000 [Veröffentlichungen der Stadtbibliothek Ulm; 20], S. 110). Einfluss und Vorbild der Leiden des jungen Werthers auf den Roman wurden in der germanistischen Forschung kontroversiell diskutiert, prinzipiell jedoch nicht in Zweifel gezogen. Beides Romane über eine unglückliche Liebe mit tragischem Ausgang, unterscheiden sie sich wesentlich in Personenkonstellation und dem Maß an Irrungen und Wirrungen, die bei Miller den Handlungsverlauf bestimmen. „Goethes Roman gehört dem individuumbezogenen Sturm und Drang an, der eine Revolution der Gesellschaft anstrebt, der `Siegwart`steht in der älteren Tradition der europäischen Empfindsamkeit, deren Ideal der Ausgleich zwischen Individuum und Gesellschaft ist« (ebd., S. 112). Und im Urteil Elschenbroichs: „Gemeinsam ist beiden Werken die Freude am Detail der bürgerlichen Alltagswelt, die Liebe zur idyllischen Natur, der Einfluß Rousseaus, die Klopstockverehrung, die Nähe zum Autobiographischen, freilich erscheint dies alles bei M[iller] einem Trivialisierungsvorgang unterworfen, der mit dem Rückgriff auf Richardsonsche Tugendideale das Rührende an die Stelle des Erschütternden setzte und so bei aller Gefühlsseligkeit letztlich einer predigerhaften Durchschnittsmoral das Feld überließ. Trotz zwiespältiger Bewertung des Klosterlebens hat `Siegwart` der katholisierenden Verklärung des Mönchtums in der Romantik den Weg bereitet« (Adalbert Elschenbroich: Miller, Johann Martin. In: Neue deutsche Biographie. Hg. von der Historischen Kommission bei der Bayerischen Akademie der Wissenschaften. 17. Band. Berlin: Duncker \& Humblot 1994, S. 514-516, hier: S. 515). 
bin verunreinigt! Bin ein armer Mann! [...] Der Jude war nicht mehr zu bewegen, in die Kutsche zu sitzen. Er setzte sich von aussen hin, ungeachtet es heftig regnete. Auf der Station $\mathrm{a} ß$ der Jude nichts als trockenes ungesäuertes Brod, das er bey sich hatte, weil der Jude nichts von Christen Zubereitetes geniessen darf. Siegwart bedaurte recht von Herzen das Schicksal dieser armen Leute, und sah den Juden oft mitleidig von der Seite an, der zuweilen bey sich selbst seufzte. ${ }^{65}$

Die Zusammenführung Angehöriger unterschiedlicher Stände und Bevölkerungsgruppen im abgeschlossenen Raum einer Postkutsche ist ein gängiges Motiv in der Romanliteratur der 70er und 80er Jahre des 18. Jahrhunderts, das sich in der literarischen Auseinandersetzung mit Juden besonders bewährt hat. ${ }^{66} \mathrm{Im}$ vorliegenden Beispiel treffen ganz klare Gegensätze aufeinander: der zartfühlende Siegwart, der zaghaft und ohne jeden Erfolg den Juden in Schutz zu nehmen sucht und Mitleid empfindet, kontrastiert mit dem ungehobelten und derben Fahrer ebenso wie mit dem überheblichen und aufdringlichen Offizier. Beiden ermangelt es, wie in weiteren Episoden exemplifiziert, an grundlegenden Umgangsformen - oder um mit dem späteren, klassischen Begriff zu sprechen: an Herzensbildung. Am deutlichsten zeigt das aber der Mutwille, mit dem sie den ihnen ausgelieferten Fahrgast drangsalieren - den »armen « Juden. ${ }^{67}$

Die literarische Gestaltung der Judenfigur ist äußerst dürftig; sie erschöpft sich in der schablonenhaften Reproduktion gängiger Klischeevorstellungen, weil ihre Entfaltung ins Individuelle weder von Zweck noch sonst von Interesse ist.

Ins Visier tritt der Jude nur dann, wenn er Siegwarts Aufmerksamkeit erregt, durch den die Erzählung - erzähltheoretisch gesprochen - fokalisiert ist. Die Wahrnehmung des Juden entspricht also der seinen; voreingenommen registriert sie das, was der eigenen Erwartungshaltung entspricht und durch die Abweichung vom Vertrauten, also durch seine Fremdheit, dazu angetan ist, den Blick des Betrachters auf sich zu ziehen. Dazu gehört die vom Juden geäußerte Furcht vor Verunreinigung, der Verzehr des »ungesäuerte[n] Brod[es] « ${ }^{68}$ ebenso wie die Verrichtung des Abendgebets. ${ }^{69}$ Der traditionell-christliche Topos von der

65 Johann Martin Miller: Siegwart. Eine Klostergeschichte. Zweyter Theil. Leipzig: Weygand 1776, S. 538-539.

66 Zum Motiv der »christlich-jüdischen Reisegesellschaft« s. Gunnar Och: Imago judaica. Juden und Judentum im Spiegel der deutschen Literatur 1750-1812. Würzburg: Königshausen \& Neumann 1995, S. 128.

67 »Die betreffende Passage gehört freilich zu den gelungeneren Partien des Romans, d. h. zu jenen detailreichen Genrebildern, mit denen der Verfasser das soziale Leben seiner Zeit einzufangen versucht« (ebd., S. 130).

68 Miller, Siegwart (wie Anm. 65), S. 539.

69 Ebd., S. 543. 
Abgesondertheit und Halsstarrigkeit der Juden (Speise- und Reinheitsvorschriften) klingt hier an, allerdings nicht vorwurfsvoll, sondern erregt, durch Perspektive und nüchternen Ton der Erzählung gemildert, Bedauern und Mitleid. Die hergebracht christliche Sichtweise wird von der Deutungstradition der Aufklärung überlagert, die hier in ihrer gefühlsbetont (sentimentalen) Version in Erscheinung tritt: der Jude als rückständiger, unterdrückter Außenseiter, dem es an Bildung mangelt, um sich aus dieser Position zu befreien. Im Seufzen des »armen Juden « ist die ganze Ausweglosigkeit einer rechtlosen Randexistenz aufgehoben, die, sobald sie einen Schritt aus ihrer Isolation herauswagt, selbst dem Aufgeklärten und Empfindsamen lästig und widerwärtig erscheint. So heißt es an einer weiteren Stelle:

Der Jude, der die Geschwätzigkeit mit seiner ganzen Nation im hohen Grad gemein hatte, plauderte beständig mit dem jungen Kaspar; erzählte ihm alle seine Familienumstände, daß er einen Sohn habe, der so alt sey wie er; daß ihm seine Rebekka vor zwey Jahren gestorben sey u. s. w. Seine Neugierde wollte aus Kaspar eine gleiche Vertraulichkeit herauslocken; aber dieser sagte immer nur: So! und Ja, und Nein. ${ }^{70}$

Neben der hinlänglich demonstrierten Gesetzestreue oder »Frömmigkeit« des Juden zählt zu dessen Charaktereigenschaften auch eine Mitteilsamkeit, die, abschätzig als »Geschwätzigkeit« bezeichnet, jeglichen Anspruch auf Sinnhaftigkeit und Relevanz einer sprachlichen Äußerung verwirkt. ${ }^{71}$ Der aufgeklärte Erzähler lässt den Juden nur andeutungsweise zu Wort kommen. Was ihm als literarischer Gestalt Kontur verleihen könnte, nämlich biographische Zusammenhänge unter Verweis auf Herkunft und familiäre Bande, wird nicht nur unterbunden, sondern auch so inszeniert, dass das Desinteresse berechtigt erscheint. Kein Gehör schenkt dem Juden ein neu zugestiegener junger Mann, dem der Abschied von der Familie deutlich zugesetzt hat. Mit Kaspar, dem Studenten in spe, betritt

70 Ebd., S. 541-542.

71 Schudt hatte in seiner judenfeindlichen Polemik die Juden folgendermaßen charakterisiert: »Die Kennzeichen des Gemüths der Juden sind / ein innerlich auffgeblasener Sinn / darbey doch falsch-freundlich / schmeichelhafft / schwätzhafft / wo es die Noth oder ihr Nutzen erfordert / demüthig / allezeit aber tückisch und betrüglich / ruhmräthig und Gewinnsüchtig \& darzu kommt der sonderliche Accent oder Pronunciation und Aussprach der Rede / daß ein Jud / so bald er nur den Mund auffthut / verrathen ist / dann seine Sprach verräth ihn « (Johann Jacob Schudt: Jüdische Merckwürdigkeiten. Vorstellende was sich Curieuses und denckwürdiges in den neuern Zeiten bey einigen Jahr-hunderten mit denen in alle IV. Teile der Welt / sonderlich durch Teutschland / zerstreuten Juden zugetragen. Sammt einer vollständigen Franckfurter Juden-Chronik [...]. Teile 1-3. Frankfurt, Leipzig: Samuel Tobias Hocker, 1714-1717, VI. Buch, 21. Cap., § 31, S. 369). 
der verhätschelte Sohn eines Amtmanns den Schauplatz und fügt dem Abbild von der Gesellschaft im Kleinen ein weiteres Element hinzu: des bildungsbeflissenen Bürgertums. Während die literarische Gestaltung der Personen generell am Exemplarischen ausgerichtet ist, Kondukteur und Offizier ebenso als Vertreter je der einfachen, nicht-bäuerlichen Landbevölkerung und des Offizierskorps bzw. niederen Adels aufzufassen sind, ist der Jude in der Darstellung, ganz auf den Träger »nationaler« Eigenschaften reduziert, zum Typus verflacht. Es ist die literarisch-ästhetische Entsprechung der Wahrnehmung von Juden als distinkter Gruppe, deren Andersartigkeit, einst religiös motiviert, national und wesenhaft gedeutet wird. In der Interaktion mit Vertretern unterschiedlicher Stände eröffnet sich eine zweite Perspektive auf den Juden, der nur bei Siegwart auf Mitleid, bei allen anderen aber auf Ablehnung stößt.

So stereotyp die Zeichnung von Juden in der Literatur des 18. Jahrhunderts auch gewesen sein mag, lässt sich doch eine Differenzierung nach Funktion und sittlichem Verhalten erkennen. 1749 hatte Lessing sein Lustspiel Die Juden verfasst, 1747/8 Gellert seinen Roman Das Leben der Schwedischen Gräfin von $G^{* * *}$ veröffentlicht; die Figur des »edlen (deutschen) Juden« war geschaffen, das Modell, nach dem sich die Mehrheit der Aufklärungsromane ausrichtete. ${ }^{72}$ Der darin zum Ausdruck kommende Toleranzgedanke birgt in sich eine Assimilationserwartung, deren Erfüllung den Juden erst zu dem sittlichen Menschen macht, als der er in Erscheinung tritt. Sein Judentum hat er als sozialen Habitus längst zurückgelassen und gegen den des aufgeklärten, über jegliche konfessionelle Zuschrei-

72 Gutsche weist in ihrer Studie zur Konstruktion des Jüdischen in der Literatur des 17. Jahrhunderts darauf hin, dass es positiv gezeichnete Judenfiguren durchaus schon vor Lessing und Gellert gab, namentlich in Eberhard Werner Happels Der Asiatische Onogambo (1673) oder Jean Baptiste de Chevremonts Der georgianischen Kemiski und ihres Gemahls Alekes seltsame Liebesgeschichte (1698) (Victoria Luise Gutsche: Zwischen Abgrenzung und Annäherung. Konstruktionen des Jüdischen in der Literatur des 17. Jahrhunderts. Berlin, Boston: De Gruyter 2014 [Studien und Dokumente zur deutschen Literatur und Kultur im europäischen Kontext; 186], S. 184, 259ff.). Allerdings handelt es sich bei diesen Figuren, anders als in der Aufklärungsliteratur des 18. Jahrhunderts, nicht um europäische oder gar deutsche, sondern in einen fremden Kulturraum entrückte »orientalische« / asiatische Juden. Für die weitgehend von einem stereotyp negativen Judenbild geprägte Literatur des 17. Jahrhunderts gelangt Gutsche, die sich in ihrer Darstellung um ein nuanciertes Bild und die Aufdeckung alternativer Lesarten bemüht, zu folgendem Schluss: »Je weiter sich der Autor von seinem Text durch Fiktionalisierungsstrategien entfernt, desto unproblematischer scheint es zu sein, Figurationen des Jüdischen vorzustellen, die später positiv gezeichneten Figuren des achtzehnten Jahrhunderts ähneln. Der exotische bzw. utopische Ort bildet mithin die Voraussetzung für die Erprobung alternativer Entwürfe >des Juden« (ebd., S. 388). 
bung erhabenen Universalmenschen getauscht. So urteilt Ernst Simon über den mysteriösen Reisenden aus Lessings Lustspiel:

Im übrigen gehört er genau zu dem Typ, >dem man gar nichts anmerkt‘: er ist in der glücklichsten Weise an den gebildeten Durchschnittsbürger des 18. Jahrhunderts assimiliert; schwärmt mit Rousseau für die Natur und eifert gegen die Stadt, führt, als Merkmal seiner hohen Kultur, Bücher mit sich [...]. ${ }^{73}$

Ein Jude rein der Herkunft und möglicherweise noch dem Namen nach entsprach allerdings kaum der Lebenswirklichkeit des 18. Jahrhunderts, sondern gab vielmehr das Programm für eine Zukunft vor, in der Religion und Ethik austauschbar geworden sein sollten.

Eine ältere, weit in voraufklärerische Zeiten zurückreichende Tradition der Reduktion auf stereotyp jüdische Charaktereigenschaften bestand nach dem Paradigmenwechsel weiterhin fort: die burleske Judenfigur, ein Erbe des Stegreifspiels der Wanderbühnen und komisches Rollenfach. ${ }^{74}$ Als Hausierer oder »Schacherjuden « und ihres jüdischen Idioms wegen verlacht, entsprachen sie dem negativen Grundschema des »Bösewichts «. ${ }^{75}$

Dass der »komische Jude« nicht ausschließlich performativ realisiert, sondern auch in Prosatexten inszeniert werden konnte, belegt etwa der dritte Band der Satire-Reihe Die redende Thiere über menschliche Fehler und Laster (1740). ${ }^{76}$

73 Ernst Simon: Lessing und die jüdische Geschichte. In: Jüdische Rundschau 34 (1929), Nr 6, S. 1.

74 Och, Imago judaica (wie Anm. 66), S. 50ff., $89 \mathrm{ff}$.

75 Ebd., S. 93.

76 Die redende Thiere über menschliche Fehler und Laster. Bey ruhigen Stunden lustig und nützlich zu lesen. Drittes Gespräch zwischen einem Bär und einer Grille. Franckfurt und Leipzig 1740. - Als Verfasser zumindest eines Teils der anonym erschienenen, 43 Bände umfassenden Reihe wird der Nürnberger Rechtsanwalt, Schriftsteller und Journalist Christoph Gottlieb Richter (1717-1774) genannt. »Richter war, was ich in den gedruckten und handschriftlichen Nachrichten von ihm bezeugt finde, ein philosophischer und witziger Kopf, ein guter Jurist, und geschickter Sachwalter, ein thätiger, arbeitsamer, und mit Leichtigkeit arbeitender Mann. Aber, nach einer vernachlässigten Erziehung, und bei einem Temperamente, dem kein männlicher und kein religiöser Sinn einen Damm setzte, verleiteten ihn Leichtsinn zu allerhand Ausschweifungen, Witz und Dichtergabe zur Satyre und zum Pasquill, Bedürfniß und Mangel zur Rabulisterey, und theils zu vielen sehr unfeinen Handlungen, theils zu manchen groben Betrügereien « (Clemens Alois Baader: Lexikon verstorbener baierischer Schriftsteller des achtzehnten und neunzehnten Jahrhunderts. Bd 2,2: R-Z. Augsburg, Leipzig: Jenisch und Stage 1825, S. 25-29, hier: S. 26f.). Richters literarisches Schaffen umfasst auch die Übersetzung und Autorschaft pseudo-jüdischer Chroniken, in denen die historischen Ereignisse des 18. Jahrhunderts im biblischen Stil 
Im Gespräch mit der Grille beruft sich der Bär zum Beweis seiner Tugendhaftigkeit (»Frömmigkeit«) u. a. auf zwei Begegnungen mit Juden: Um die Aufmerksamkeit seiner Wärter auf sich zu lenken, bricht der ausgehungerte, in einem »kleine[n] Hof bey einem Gefängnuß-Thurn « gehaltene Bär in eine der Zellen ein, wo »ein Jude wegen vielen verübten, und überzeigten Betrügereyen gefangen ${ }^{77}$ gehalten wird.

Der Jude, welcher schon alle verstorbene Alt-Väter um Hülffe angeruffen hatte, machte ein solches Geschrey, daß mir die Ohren davon wehe thaten, ich aber brummte immer stärcker zu dem Fenster nein, und arbeitete beständig mehr und mehr Steine aus dem Fenster, dadurch endlich das Loch so groß wurde, daß ich mit allen beyden Pratzen zu gleich ganz bequem den Kopf in das Loch stecken, und in das Gefängnuß sehen kunte. Ob ich nun wohl keinen Appetit hatte, Jüdisches Fleisch zu fressen, so stellte ich mich doch sehr begierig bald eine Mahlzeit zu halten. In dieser Angst ergriffe der Jude von denen nunter gefallenen Steinen ein grosses Stücke, und schluge unabläßig an die Gefängnuß-Thüre, biß endlich das starcke Schlagen die vorbey gehende Menschen hinlockete, die solches andeuteten, und als man die Thüre eröffnete, und mich mit dem halben Leibe in dem gemachten Fenster-Loch stehen sahe, entstunden darüber nicht ein geringes Gelächter, man sperrte den Juden in einander Behältnuß, und ich bekam meine gewöhnliche Portion, weil man sich erst auf mich besonnen hatte. [...]

Grille: Den Juden hätte ich in seiner Angst sehen und hören mögen, weil ihm diese unverhoffte Besuchung sehr wenig Trost gebracht hat. [... $]^{78}$

Der inszenierte Einbruch ohne Absicht, dem Juden körperlichen Schaden zuzufügen, entringt den herbeigeeilten Passanten ein schadenfrohes und spöttisches Lachen. Zu komisch, weil in seiner Verächtlichkeit nicht anders wahrnehmbar, wirkt die lautstark bekundete Todesangst des überrumpelten Juden, die angesichts der ausgewiesenen Harmlosigkeit des Tieres als maßlos übertrieben erscheint. Wie im Theater bedarf es der Reaktion des Publikums, um die beabsichtigt komische Wirkung der Szene auf den Leser nicht zu verfehlen.

Zur allgemeinen Erheiterung gereicht an anderer Stelle ebenfalls das »Geschrey« eines böhmischen Landjuden, dem der Bär aus Vergeltung für dessen Schikanen übel mitspielt.

Es kamen ein paar Land-Juden als sogenannte Haußirer mit unterschiedlichen Sachen zu meinem Herrn, bey ihm etwas zu verkaufen; unter der Zeit als der erste bey meinem Herrn handelte, so kam der andere zu mir / und wolte mich foppen. Ich schaute ihm eine gute

dargestellt werden. S. dazu: Ivo Cerman: Maria Theresia in the Mirror of Contemporary Mock Jewish Chronicles. In: Judaica Bohemiae 38 (2002), S. 5-47.

77 Die redende Thiere. Drittes Gespräch (wie Anm. 76), S. 112.

78 Ebd., S. 113-114. 
Weile zu, als er mir dem Possen aber zu grob machte, so nahm ich meinem Vortheil in acht, und weil er mir als einem gedultigen Bären wohl trauete, und sich mir starck näherte, so erwischte ich ihn mit beyden Vorder-Pratzen, und trug ihn biß zu meiner Säule. Das Geschrey, so er machte, lockte alle Menschen, so auch meinem Herrn dazu, ich aber machte meine Lust bald mit ihm aus, denn sonsten würden wohl manche gedacht haben, ich wollte ihm grob begegnen. Derowegen risse ich ihm nur den blau-gesterckten Kragen, oder das so genannte Kreß vom Halse, gab ihm ein paar mittelmäßige Ohrfeigen, oder Maulschellen, rauffte ihm ein wenig bey seinem Barte, doch risse ich ihm keine Haare aus. Nach diesem nahm ich seinen Hut, setzte solchen auf, und nahm zugleich das Kreß um meinen Hals, darüber alle Zusehende lachten, und den Juden ließ ich ohne den geringsten Schaden wieder gehen. ${ }^{79}$

Der traktierte Jude, durch Beruf und Kleidung äußerlich diskriminiert, wird mit dem Mittel der Travestie dem Gespött der Umstehenden preisgegeben: War die altmodische Tracht, die in Böhmen vorschriftsmäßig zum Zweck der Kennzeichnung getragen werden musste, ${ }^{80}$ schon Demütigung genug, so wirkt sie, vom Bären zur Maskerade angelegt, regelrecht als Verhöhnung der menschlichen Natur des Juden. In der Darstellung scheinen dessen menschliche Qualitäten gemessen an Tugenden wie Selbstreflexion und -disziplin reduziert, wie Juden so oft ein unbeherrschtes, triebhaftes Verhalten unterstellt wurde $-{ }^{81}$ und das im Gegensatz zum Bären, der geduldig und wohlbedacht seine körperliche Überlegenheit demonstriert, in der Absicht, seinen Bedränger in die Schranken zu weisen. Der

79 Ebd., S. 120-121.

80 Zur Halskrause, dem sog. »Kröss« oder »Kress«, s. Tobias Jakobovits: Die Judenabzeichen in Böhmen. In: Jahrbuch der Gesellschaft für Geschichte der Juden in der Čechoslovakischen Republik. Hg. von Samuel Steinherz. Jg 3, 1931 (Frankfurt: Textor 2008), S. 145-184, hier: S. 154f., 170.

81 Schudt hatte in seiner ethnographischen »Studie« Jüdische Merckwürdigkeiten (1714-1718), einer säkular, d. h. nicht theologisch argumentierenden judenfeindlichen Polemik, Tier-Metaphern zur Charakterisierung jüdischer Eigenschaften verwendet, u. a. den Ungeziefer-Vergleich. "Dieser Sprachgebrauch drückt Abscheu vor und Distanz zu zahl- und namenlosen Juden aus, deren auffälligste - und abstoßendste - Züge mehr an Tiere als an Menschen erinnern. Sie reagieren auf instinktive Weise und nicht gemäß den Normen der Gesellschaft, in deren Mitte sie sich niederlassen. Sie verfügen weder über gutes Benehmen und feine Manieren noch über die vielleicht protestantischste aller Tugenden, Selbstdisziplin, die sich die christliche Gesellschaft in dem frühneuzeitlichen Verfeinerungsprozeß der Umgangsformen, den der Soziologe Norbert Elias schon 1936 beschrieben hat, angeeignet hat « (Maria Diemling: "Daß man unter so viel tausend Menschen so fort einen Juden erkennen kann«: Johann Jacob Schudt und der jüdische Körper. In: Die Frankfurter Judengasse. Jüdisches Leben in der Frühen Neuzeit. Hg. von Fritz Backhaus, Gisela Engel, Robert Liberles, Margarete Schlüter. Frankfurt a.M.: Societäts-Verlag 2006 [Schriftenreihe des Jüdischen Museums Frankfurt am Main; 9], S. 77-89, hier: S. 83). 
Jude wird hier zum Tier, während der Bär gleichermaßen Witz wie »Frömmigkeit« besitzt.

Die den beiden Judenfiguren entgegengebrachte Geringschätzung findet ihre Rechtfertigung in dem Hinweis auf moralische Mängel: der eine ausgewiesener Betrüger, der andere Tierquäler haben sie, so die inhärente Logik, nichts Besseres verdient, als betrogen und gequält $\mathrm{zu}$ werden. Dass sich die dabei freigesetzten Aggressionen allerdings gegen ihr Jude-Sein richten, lässt sich u. a. an der Fixierung auf ihre äußerlichen Kennzeichen, die sog. Judenabzeichen, ablesen und bezeugt einmal mehr die Gleichsetzung von Judentum und verdorbenem, betrügerischem Charakter.

Unter Einfluss der Aufklärung entwickelte sich in der zweiten Hälfte des 18. Jahrhunderts ein Mischtyp, in dem sich didaktischer und Unterhaltungsanspruch, parodistische Elemente und positive Tendenz miteinander verbanden. ${ }^{82}$

Mit Jenzsch und Neubauer ist für die Bühnenstücke des 18. und frühen 19. Jahrhunderts festzuhalten, dass es sich, unabhängig vom Typ, bei den meisten Judenfiguren um alleinstehende Männer fortgeschrittenen Alters handelt, die in der Regel als Händler, Trödler oder Geldverleiher auftreten. »[...] auf der Szene bleiben sie Einzelne, Fremde gegenüber dem Kontext der Mitfiguren. $\aleph^{83}$ Nur in seltenen Fällen wird »das Vorhandensein einer Familie angedeutet «. ${ }^{84}$

Gäbe es einen Idealtypus dieser Gestalt, er wäre ein bärtiger Mann jenseits der fünfzig in der traditionellen Tracht der reisenden (ost)jüdischen Händler; durch bestimmte sprachliche Muster unterscheidet er sich von den übrigen Figuren, und auch in seinem Habitus und Gebaren gibt er sich als jüdischer Noth-Händler und Trödler zu erkennen. ${ }^{85}$

Der Jude in Millers Siegwart lässt sich keinem dieser Typen zuordnen, was nicht zuletzt an den unterschiedlichen Gestaltungsmöglichkeiten der Gattungen Prosa und Drama liegt; moralisch-didaktische Funktion besitzt er im Gefüge des Romans nur insofern, als sich am Verhalten der Reisenden ein Mangel an Sittlichkeit offenbart und er gleichzeitig zum Prüfstein für Siegwarts Einfühlsamkeit wird. Auf das Motiv des Wuchers und unlauterer Handelspraktiken andererseits rekurriert der Text nicht einmal andeutungsweise, ganz $\mathrm{zu}$ schweigen von

82 Och, Imago judaica (wie Anm. 66), S. 104ff.

83 Hans-Joachim Neubauer: Judenfiguren. Drama und Theater im frühen 19. Jahrhundert. Frankfurt, New York: Campus Verlag 1994, S. 44.

84 Helmut Jenzsch: Jüdische Figuren in deutschen Bühnentexten des 18. Jahrhunderts. Eine systematische Darstellung auf dem Hintergrund der Bestrebungen zur bürgerlichen Gleichstellung der Juden, nebst einer Bibliographie nachgewiesener Bühnentexte mit Judenfiguren der Aufklärung. Hamburg (Diss.) 1971.

85 Neubauer, Judenfiguren (wie Anm. 83), S. 45. 
anderen üblen Gemeinplätzen antijudaistischen Zuschnitts. Der Funktion nach Reflektor des moralischen Verhaltens gegenüber rechtlosen Außenseitern, ist die Judenfigur ihrem Gehalt nach als »einfacher, geplagter Jude« einzuordnen. Der Ausruf »Bin ein armer Mann! $«^{86}$ ist zunächst Ausdruck des Selbstmitleids und beinhaltet die Bitte um Schonung; die darin aufgerufene Armut verweist auf die Schmähungen und Nachteile, die dem Juden aus seiner Stellung in der Gesellschaft erwachsen, von den Sonderlasten der Judensteuern angefangen bis zum Ausgeliefertsein in der Interaktion mit Christen. Sie ist aber, wie ich meine, auch wörtlich, d. h. materiell, im Sinn von »Mittellosigkeit« aufzufassen. Dafür spricht der kärgliche Proviant des Juden und, wenn man möchte, sein sorgenvolles Seufzen. Den Grund seiner Reise erfahren wir nicht; lediglich Ausgangs- und Endpunkt derselben, nämlich Günzburg und Donauwörth. Es ist wohl nicht weit gefehlt, sich als reales Vorbild einen schwäbischen Landjuden vorstellen zu wollen, in geschäftlichen Angelegenheiten unterwegs zu einem der regelmäßig in Donauwörth abgehaltenen Märkte. Das traditionsreiche Ichenhausen etwa liegt zehn Kilometer südlich von Günzburg und dem daran angrenzenden Leipheim, wo Miller »fast seine ganze Kindheit verbrachte «. ${ }^{87}$ Obwohl in Leipheim selbst zu dieser Zeit keine Juden mehr ansässig gewesen sein dürften, musste Miller die jüdischen Hausierer und Händler der umliegenden Ortschaften gekannt haben.

Die Annahme, unter der stereotypen Zeichnung des Millerschen Juden schimmere ein landjüdisches Substrat als reale Vorlage durch, lässt sich aus historischdemographischen Gründen nicht zurückweisen; sie ist aber auch nicht aussagekräftig. In voremanzipatorischer Zeit, wo gerade im Süden Deutschlands die wenigen privilegierten Juden, Hofjuden oder andere vermögende Kaufleute und Bankiers, eine geringe Minderheit innerhalb der Judenschaft darstellten, und nur vereinzelt Städte wie Frankfurt oder Fürth über größere jüdische Gemeinden verfügten, lebte der Jude, den man im wirklichen Leben antreffen konnte, auf dem Land. Differenziert wurde allerdings anhand von Status und Stellung in der Gesellschaft zwischen privilegierten, »vornehmen«, also auch reichen Juden und den traditionell lebenden, »gemeinen « Juden. Dieser Unterscheidung war der Gegensatz von Stadt und Land in gewisser Weise inhärent, aber keineswegs hinlängliches Unterscheidungskriterium, sondern Selbstverständlichkeit.

Das konzeptuelle Nahverhältnis von sozioökonomischem Stand und Siedlungsmuster erhellt aus zeitgenössischen Quellen der Epoche, in denen das Attribut »arm« ein treuer Begleiter (Kollokator) des »Landjuden« ist. Drei Beispiele

86 Miller, Siegwart (wie Anm. 65), S. 538.

87 Breitenbruch, Johann Martin Miller (wie Anm. 64), S. 14. 
unterschiedlicher Herkunft sollten zur Illustration und Stützung der Behauptung ausreichen.

In seinem »Schreiben aus Hagenau über den Kulturzustand der Israeliten in Frankreich « (1809) erörtert der Elsässer Widersheim ${ }^{88}$ auf die Frage hin, ob es unter den Juden auch Landarbeiter und Ackerbauern gäbe, die Verhältnisse folgendermaßen:

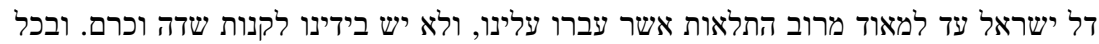

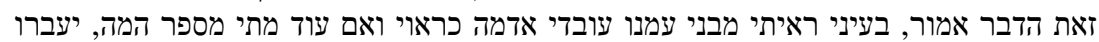

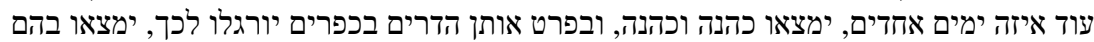
עונג וידריכו בניהם לעשות ימתדים כמותם.

Der nicht genannte Übersetzer des Briefes, möglicherweise Redaktionsmitglied der in Dessau herausgegebenen Zeitschrift, gibt den Passus frei wieder:

[...] theils auch giebt es unter den Landjuden nur wenige, die ein zum Ankauf der Aecker hinlängliches Vermögen besitzen. Lassen Sie nur noch eine kurze Zeit verstreichen, und ich zweifle nicht, daß sich auch diesem der Menschheit so nützlichen Gewerbe mehrere Israeliten, besonders unter den Landbewohnern, mit Vergnügen widmen und ihre Kinder für diesen Stand bilden werden. ${ }^{90}$

Obgleich »jisra'el« schlicht einen Juden bezeichnet, wird daraus in der stark raffenden Übertragung ein »Landjude«. Der agrarische Kontext der Stelle scheint diese Verdichtung von Ländlichkeit und Armut im Begriff »Landjude« zu rechtfertigen.

Ein zweites Beispiel ganz anderer Art integriert in das Bild der materiellen Not auch das des sozialen Elends, der Verwahrlosung. Es ist der Verteidigungsschrift aus einem Strafprozess von 1783 entnommen, in dem die geständige Dienstmagd Frommet des Mordes an ihrer Brotherrin Schönle Maas, wohnhaft in der Frankfurter Judengasse, zum Tode verurteilt worden war. Der Historiker Georg Ludwig Kriegk hatte im Rahmen seiner Tätigkeit als Stadtarchivar zu Frankfurt Vor-

88 Carmoly identifiziert den Verfasser als Nathanael ben Isaak Widersheim, einen Abgeordneten der Notablen-Versammlung zu Paris und Mitglied des Sanhedrin ([Eljakim] Carmoly: Bemerkungen zur Geschichte der Metzer Rabbinate von Tsarphati. In: Israelitische Annalen. Ein Centralblatt für Geschichte, Literatur und Cultur der Israeliten aller Zeiten und Länder, Jg 1840, H. 21, S. 185-186, hier: S. 186).

89 Widersheim der ältere: Schreiben aus Hagenau über den Kulturzustand der Israeliten in Frankreich. In: Sulamith. Zeitschrift zur Beförderung der Kultur und Humanität unter der jüdischen Nation. Hg. von David Fränkel. Deßau, Jg 2, H. 2, 1809, S. 279-292, hier: 288-289.

90 Ebd., S. 290. 
geschichte, Tathergang und anschließendes Gerichtsverfahren anhand des Aktenmaterials rekonstruiert. Nach Fällung des Todesurteils, so Kriegk, habe der Verteidiger mehrere Versuche unternommen, eine Strafmilderung zu erwirken, dabei aber einen Fehlschlag nach dem anderen erlitten. In seinen Gesuchen berief er sich unter anderem auf das jugendliche Alter Frommets, die zum Zeitpunkt der Tat erst fünfzehn oder sechzehn Jahre alt gewesen war, und auf ihre Herkunft aus einem prekären Milieu. Frommet stammte aus dem hessischen Dreieichenhain, ${ }^{91}$ hatte früh den Vater verloren und musste, der schlechten finanziellen Lage der Familie wegen, noch jung in Dienst treten. »Der Verteidiger sagte«, so Kriegk in einer Paraphrase, »die armen Landjuden würden kaum erzogen, und der Verstand bedürfe bei solchen Kreaturen zu seiner Entwicklung eine längere Zeit, als bei anderen Menschen. ${ }^{92}$ Und weiter, diesmal den Verteidiger zitierend:

Es ist bekannt [....], wie weit die Erziehung der Juden von der christlichen überhaupt verschieden ist, indem die tägliche Erfahrung lehret, daß die rohesten Sitten der jüdischen Nation ganz eigen sind. Auch diejenigen Juden, denen es an zeitlichen Glücksgütern nicht fehlet, selbsten solche, die von ihnen für die vornehmsten gehalten werden, verrathen sich gar leicht durch ihr rohes und unkultivirtes Wesen, so daß sie allein schon dadurch, wenn ihnen auch alle sonstigen Kennzeichen fehlen, die Nation, wozu sie gehören, zu erkennen geben. Um wie viel mehr muß also eine rohe und gleichsam viehische Erziehung bei solchen Landjuden herrschen, von welcher Art Inquisitin herzustammen das Unglück hat! Wer erinnert sich nicht, je einmal einen Trupp solcher elenden Geschöpfe von Landjuden, ihre Kinder und Habseligkeiten auf dem Buckel tragend, gesehen zu haben? Und wem ist bei einer solchen Erscheinung nicht sogleich der geringe Unterschied zwischen Menschen und Vieh beigefallen $?^{93}$

Angelegentlich, wie aus den Archivunterlagen hervorgeht, um seine Klientin bemüht, greift der Verteidiger zu deren Entlastung auf antijüdische Ressentiments zurück, wonach Juden, egal welchen Standes, über schlechte Umgangsformen verfügten. Die Behauptung, bei den Landjuden nehme dieser Mangel nahezu animalische Formen an, gründet auf einem »argumentum a fortiori«, nicht auf eigener Anschauung. Diese kennt die Landjuden nämlich nur aus der Entfernung, wie sie im Vorbeiziehen ein Bild des Elends bieten. Die Perspektive des Verteidigers ist eine städtisch-bürgerliche, sie registriert das Augenfällige, das auch

91 In Dreieichenhain werden Juden zum ersten Mal 1428 erwähnt, in den folgenden Jahrhunderten waren »dann immer einige Schutzjuden dort ansässig« (Paul Arnsberg: Die jüdischen Gemeinden in Hessen. Anfang, Untergang, Neubeginn. Bd 1. Frankfurt: Societäts-Verlag 1971, S. 143).

92 G[eorg] L[udwig] Kriegk: Die Judenmagd Frommet. In: Zeitschrift für deutsche Kulturgeschichte. Neue Folge. Hg. von J. H. Müller, Jg 2 (1873), S. 275-288, hier: S. 281.

93 Ebd., S. 281-282. 
außerhalb der Dörfer Sichtbare, und ist weltanschaulich durchaus auf Höhe der Zeit, wenn das Individuum als Produkt von Milieu und Erziehung begriffen wird..$^{94}$ Zum Repräsentanten des Landjudentums wird hier der vagierende Betteljude, und im Appell an das Erinnerungsvermögen zeigt sich das Kollektive der Wahrnehmung.

Sieht man einmal vom diffamierenden Ton der Zeilen ab, deren Krassheit wohl auch Kalkül gewesen sein mag, den Richter im Sinn der Verteidigung zu beeinflussen, war die Evokation von Armut und Elend bei Weitem nicht gefehlt. Auch der »Würzburger Domkapitular, Stadtschuldirektor und Professor für Dogmatik und Polemik « ${ }^{95}$ Franz Oberthür (1745-1831) zählt zu den Hemmnissen einer sofortigen rechtlichen Gleichstellung der Juden, die es wohlüberlegt vorzubereiten heiße, »die große Anzahl und die höchst traurige Lage der mit uns in so nahen Verhältnissen stehenden Judenschaft in ritterschaftlichen, unmittelbaren, freyen Ortschaften und Schlössern, die sich allein, in dem einzigen Canton Röhn und Werra, wenigstens auf 1200 beläuft«. ${ }^{96}$ Denn, fährt er fort:

Mehr auf eigenen Gewinn, als an Nachbarschaft und Menschheit denkende freye Reichsritter, tragen kein Bedenken, in ihre Ortschaften und einzelne freye Besitzungen, die sie in fremden Ortschaften haben, so viele Juden aufzunehmen, als der Raum nur immer zuläßt, wo sodann zahlreiche Familien so enge beysammen wohnen, daß es ein Wunder ist, wenn nicht mehrere Krankheiten von solchen Hütten des Elends ausbrechen, und weit um sich herum Verwüstungen anrichten. Der Gutsherr ist zufrieden, wenn er Schutzgeld bekommt, und überläßt es seinen Schutzverwandten, wo und wie sie das Geld, welches sie ihm entrichten müssen, und was sie sonst noch für ihre eigene Familie bedürfen, auftreiben mögen. Sie wuchern dann, so weit und breit es angehen mag, im Lande herum, setzen die Judenschaft in Mißcredit, und vervielfältigen sich weit über alles Verhältniß zu den Nahrungs- und Erwerbungsmitteln hinaus. [... $]^{97}$

94 Bereits Schudt hatte in seinen Jüdischen Merckwürdigkeiten die »Unsauberkeit« und den angeblich »üblen Geruch« als Kennzeichen der »Polnischen und Teutschen« Juden definiert, ebenso wie den Mangel an Selbstdisziplin, ihr »tierisches « Wesen, sozial abgeleitet. - «[Schudt] unterscheidet sehr klar zwischen den abstoßenden Erscheinungen aschkenasischer Juden und den weitaus gepflegter auftretenden sephardischen Juden, die er oft in Hamburg und Altona getroffen hatte« (Diemling, »Daß man unter so viel tausend Menschen so fort einen Juden erkennen kann « [wie Anm. 81], S. 81f.).

95 Michael Langer: Zwischen Vorurteil und Aggression. Zum Judenbild in der deutschsprachigen katholischen Volksbildung des 19. Jahrhunderts. Freiburg, Basel, Wien: Herder 1994 (Reihe »Lernprozeß Christen Juden«; 9), S. 152.

96 Franz Oberthür: Vorrede. In: Des Flavius Josephus Selbstbiographie. Uebersetzt und mit Anmerkungen versehen von J. B. Frise. Altona: Johann Friedrich Hammerich 1806, S. 1-44, hier: S. 19.

97 Ebd., S. $19 f$. 
Ein weiteres Hindernis sah Oberthür in den aus Armut zum Vagabundieren gezwungenen Juden; dass

[...] viele einzelne Juden, und ganze jüdische Familien, ohne Heimat, ohne Vaterland, ohne Gewerbe von einem Ort zum andern herumirren, der angesessenen Judenschaft als Bettler von Profession höchst lästig fallen, das Land mit Taugenichtsen bevölkern, Kinder zeugen, ohne sie zu etwas anderm erziehen können, als zu eben dieser herumstreifenden Lebensart, die auch ihre Eltern trieben, und welche die jüdische Nation verächtlich machen muß.98

Für das späte 17. und 18. Jahrhundert ist ein allgemeines Bevölkerungswachstum zu verzeichnen, das bei gleichbleibenden ökonomischen Kapazitäten eine Verarmung der unteren Schichten nach sich zog. In ihren wirtschaftlichen und rechtlichen Möglichkeiten von jeher stark eingeschränkt, konnten die finanziell ohnehin kaum abgesicherten jüdischen Hausierer und Gelegenheitshändler kein Auskommen finden und waren zum Betteln gezwungen. Neben den vergleichsweise wenigen ortsansässigen Gemeindebettlern existierte eine große Zahl vagierender Bettler, die in Gruppen von einer Gemeinde zur nächsten zogen. Ein Nachtlager und Verpflegung fanden sie nicht mehr, wie traditionell üblich, in den Häusern der Gemeindemitglieder, sondern in eigens für sie eingerichteten, außerhalb der jüdischen Siedlungen gelegenen Herbergen. ${ }^{99}$ Der Anteil der vollständig verarmten und auf Fürsorge und Spenden angewiesenen Juden an der jüdischen Gesamtbevölkerung lässt sich nicht rekonstruieren und auch nur schwer einschätzen: In den Regesten der jüdischen Gemeinden fanden sie, da nicht steuerpflichtig, keine Erwähnung, und anderweitig aktenkundig wurden sie nur, wenn sie in Konflikt mit Recht und Ordnung kamen. Folgt man den gelegentlich für einzelne Ortschaften publizierten Daten, so scheint die Zahl der vagierenden Bettler gegen Ende des 18. Jahrhunderts drastisch angestiegen zu sein. ${ }^{100}$ Noch Jahrzehnte später äußerte der Abgeordnete Ludwig Braun in einer

98 Ebd., S. 21.

99 Rudolf Glanz: Geschichte des niederen jüdischen Volkes in Deutschland. Eine Studie über historisches Gaunertum, Bettelwesen und Vagantentum. New York: Waldon Press 1968, S. 129ff. 100 Das Reichsdorf Gochsheim (Unterfranken) zählte um 179026 »udenhaushaltungen«. Obwohl an »keiner der gangbarsten Landstraßen « (S. 438) gelegen, wurde es im Laufe eines Jahres von rund 1200 Bettlern und Bettlerinnen aufgesucht, die von der jüdischen Gemeinde versorgt werden konnten, und weiteren 300, für die die vorgesehene Armenversorgung nicht mehr ausreichte. Innerhalb eines Zeitraums von 30-40 Jahren hatte sich nach Angabe des anonymen Verfassers die Zahl der Bettler verdoppelt und überstieg bei Weitem die Kapazitäten der jüdischen Gemeinde (Authentische Berechnung, was eine Judengemeinde von 26 Haushaltungen [im Reichsdorfe Gochsheim] jährlich zum Unterhalt ihrer bettelnden Glaubensgenossen beytragen muß. In: Journal von und für Franken 1 [1790], H. 4, S. 435-446). - Für Harburg ist eine Zahl von 
Verhandlung der zweiten Kammer der Landstände des Großherzogtums Hessen seine Besorgnis über den Zustand der »immer mehr verarmenden Landjuden «. ${ }^{101}$

\section{Zum Begriff »Landjude» - Eine Klärung aus historischer Sicht}

Die Frage, wieviel Landjüdisches in dem Millerschen Juden steckt, wirkt fehl am Platz; zum einen besitzt die Figur nicht genug Konsistenz, um über die Feststellung von Schlichtheit, Traditionsgebundenheit und Armut hinaus eine vertiefende Diskussion anzuregen, und zweitens schwingt in der Fragestellung selbst eine anachronistische Erwartung mit, die den Landjuden in Abhängigkeit von einem säkularen oder zumindest akkulturierten (Groß)stadtjuden all die Eigenschaften zuzuordnen versucht ist, die in voremanzipatorischer Zeit kennzeichnend für die entscheidende Mehrheit der (süd)deutschen Juden war. Immerhin befinden wir uns 1776 in einer Epoche, wo Bürgerrechte zwar als aufklärerische Ideen in einschlägigen Schriften existierten, aber weit von der politischen Realität entfernt waren.

Und doch: die Landjuden gab es. Nicht als das soziokulturelle Phänomen, zu dem sie im Zuge der immer rasanter voranschreitenden Verstädterung gegen Ende des 19. Jahrhunderts im wahrsten Sinn des Wortes marginalisiert und stilisiert wurden, aber als historisches durchaus. Annette Weber geht soweit, eine ganze, in ihrer kulturellen und religiösen Bedeutung weitgehend unterschätzte Epoche deutsch-jüdischer Geschichte als wesentlich vom Landjudentum geprägt zu charakterisieren: die Zeit vom 16. bis zum frühen 19. Jahrhundert. Seit dem Dreißigjährigen Krieg hätten gerade die Landgemeinden »neue, oft regional verankerte Lebensformen« entwickelt, »die sowohl Elemente der bäuerlichen als auch der höfischen Kultur aufnahmen, wobei sie aber ihre eigenständigen religiösen Riten beibehielten«. ${ }^{102}$

700-800 Betteljuden bezeugt, die 1721 im Verlauf von vier Monaten das Städtchen aufsuchten (Glanz, Geschichte des niederen jüdischen Volkes [wie Anm. 99], S. 130).

101 Verhandlungen in der zweiten Kammer der Landstände des Großherzogthums Hessen im Jahre 1821. Von ihr selbst amtlich herausgegeben. Bd 7, H. 19. und 20. Darmstadt, Giessen: Heyer und Leske 1821, S. 69. - Dass der Antisemit Hartwig von Hundt-Radowsky in seinem Judenspiegel von »Land- und Betteljuden, die mit Krätze und Ungeziefer unsere Länder besetzen, wie ihre Vorfahren Egypten« (Hartwig von Hundt-Radowsky: Judenspiegel. Ein Schand- und Sittengemälde alter und neuer Zeit. Reutlingen: I. N. Enßin'sche Buchhandlung 1821, S. 20) spricht, gibt unter anderem Auskunft darüber, wie aus antisemitischer Sicht eine undifferenzierte Gleichsetzung der beiden Gruppen sich wirtschaftspolitisch instrumentalisieren ließ.

102 Annette Weber: Ein Dokument jüdischer Heimatkunde aus Bayern. Zur Entstehung und Bedeutung der Fotosammlung Theodor Harburgers. In: Die Inventarisation jüdischer Kunst- und 
Ein skizzenhafter Abriss über die semantische Dimension des Begriffs »Landjude« ermöglicht eine differenzierte Sicht auf die Genese des Phänomens und soll Aufschluss über die Entstehung des Landjudentums im Bewusstsein des Betrachters geben.

Bis weit ins 18. Jahrhundert bezeichnete der Begriff »Landjude« primär einen rechtlichen Status, nämlich die Angehörigkeit zu einer »Landjudenschaft«. Um dem Niedergang und der befürchteten Auflösung religiöser Infrastrukturen und jüdischer Identität nach der Zerstörung eingesessener städtischer Gemeinden und der Zerstreuung der Juden über unzählige Dörfer und Kleinstädte entgegenzuwirken, wurden im 16. und 17. Jahrhundert Vereinigungen zur autonomen Verwaltung jüdischer Belange ins Leben gerufen, deren Gründung zum Zweck der kollektiven Besteuerung von der staatlichen Obrigkeit befürwortet wurde. Diese als »Landjudenschaften« bezeichneten Körperschaften stellten einen »Gesamtverband aller Juden eines Herrschaftsgebietes, die das Wohnrecht besaßen, d. h. aller ১Schutzjuden«" dar. "Sie gehörten dem Verband unmittelbar, nicht über eine Ortsgemeinde an. ${ }^{103}$ Landjudenschaften waren somit »keine Gemeindeverbände, sondern Vereinigungen von Individuen «. ${ }^{104}$ »Größere und vermögende Gemeinden standen [...] meist außerhalb der angrenzenden Landjudenschaft, oder sie entwickelten allmählich, besonders in Süddeutschland, eine Sonderstellung innerhalb der Landjudenschaft, wie z. B. Mannheim oder Fürth. « ${ }^{105}$ Die Bezeichnung »Landjudenschaft « leitet sich, wie u. a. aus dem hebräischen terminus technicus « קהל מדינה, בני המדינה « ersichtlich, vom Begriff»Land» in der Bedeutung von »Herrschaftsgebiet « und nicht etwa der mit dem ländlichen Raum assoziierten Siedlungsform des Dorfes her. "Landjude« im rechtlichen Sinn konnte demzufolge auch ein »Stadtjude « sein. ${ }^{106}$ Die Abhängigkeit des Begriffs vom Territorium im politischen Sinn verdeutlicht die attributiv beigefügte spezifische

Kulturdenkmäler in Bayern. Bd 1. Hg. von den Central Archives for the History of the Jewish People, Jerusalem und dem Jüdischen Museum Franken. Fürth: Jüdisches Museum Franken 1998, S. 23-41, hier: S. 24.

103 Breuer, Frühe Neuzeit und Beginn der Moderne (wie Anm. 7), S. 188.

104 Ebd., S. 189.

105 Ebd., S. 190.

106 Zur eindeutigen Abgrenzung von der jüdischen Bevölkerung der Residenzstadt Susa gibt Michaelis in seiner Übertragung des Esther-Buches den Begriff» « (Esther 9,19) mit »Dorfjuden« wieder (Johann David Michaelis: Deutsche Übersetzung des Alten Testaments, mit Anmerkungen für Ungelehrte. 13. Teil: Esra, Nehemia und Esther. Göttingen: Vandenhoeck 1783, S. 93). 
Zugehörigkeit zu einem solchen, wenn beispielsweise von "Anspachischen Land-Juden $\aleph^{107}$ die Rede ist.

Nichtsdestoweniger wiesen der »Landjude« als Bezeichnung eines rechtlichen Status und der im Verband kleiner ländlicher Gemeinden lebende Jude naturgemäß eine hohe Kongruenz auf, aus der sich die zweite, ursprünglich sekundäre Bedeutung des Begriffs ergab. Kiessling schildert in seiner Monographie zu den Forchheimer Juden den Fall eines gewissen Lazarus Löw aus Weilersbach, der sich 1762 »um eine Schutzstelle in dem unweit von Forchheim gelegenen Dorf« bemühte. Der zuständige Kastner von Forchheim, zu dessen Amtsbereich die Ortschaft gehörte, meldete nach Bamberg, dass die »zu [...] Waillersbach sich befindlichen Land-Juden in der jährlichen Nahrung und Handelsschaft sowohl, als auch dem Vermögen nach sehr viel gegen denen Vorchheimer Stadt-Juden differirten und fast um die Helfte diesen unterliegeten.« Daher könne der Bittsteller das den Stadtjuden auferlegte »Vermögensquantum nur zur Helfte ganz verläßig beybringen. ${ }^{108}$ Die in Weilersbach ansässigen Juden zählen ebenso wie die Forchheimer zum Verband der Bambergischen Landjuden, werden hier allerdings im Gegensatz zu den "Stadtjuden« als "Land-« im Sinn von "Dorfjuden« apostrophiert. Der »Landjude« tritt hier in seiner doppelten Bedeutung auf, weil er beide Perspektiven in einem Begriff integriert, die obrigkeitlich-territoriale und in Abhängigkeit des Landes von der Stadt, nämlich als Umland und sozialer Status - als Peripherie.

Die rezente Forschung, an den Kriterien Ortsgröße (Einwohnerzahl), Siedlungsund Wirtschaftsform ausgerichtet, klassifiziert Forchheim mit seinen rund 4.400 Einwohnern (1880), darunter über 200 Juden, als »Landgemeinde ${ }^{109}$ Abgesehen von den sozioökonomischen Faktoren zeichne sich eine »Landgemeinde« durch ihr Traditionsbewusstsein aus, das anders als im städtischen Kontext weder reflektiert noch ideologisch gerechtfertigt werden musste. Diese Vorstellung steht wesentlich unter dem Einfluss der demographischen Umwälzungen der Emanzi-

107 Wahrhafte Species Facti über die, von fünf Hochfürstlich Anspachischen Land-Juden, mit einem jungen minderjährigen Bauern-Bursch, getriebene verabscheuenswürdigste Betrügereyen und Wucher-Streich. [1771]. - Die in der antijüdischen »Entdeckungsschrift« aufgeführten Juden stammen aus Dittenheim, Gunzenhausen und Heidenheim.

108 Rolf Kilian Kiessling: Juden in Forchheim. 300 Jahre jüdisches Leben in einer kleinen fränkischen Stadt. Forchheim: Verlag Kulturamt des Landkreises Forchheim 2004, S. 16 (unter Bezug auf Staatsarchiv Bamberg B 53, 341, 4.5).

109 Pinkas Hakehillot. Encyclopedia of Jewish Communities from Their Foundation till after the Holocaust. Germany. Vol. 1: Bavaria. Ed. by Baruch Zvi Ophir. Jerusalem: Yad Vashem 1972, S. 243. 
pations- und Kaiserzeit, in deren Folge sich die jüdischen Zentren in die großen Ballungsräume verlagerten, und die verbleibenden (tendenziell traditionellen) Kleingemeinden pauschal unter dem Begriff »Landgemeinden « subsumiert wurden. Auf frühere Epochen angewandt, täuscht der Einheitlichkeit suggerierende Begriff über eine innere Differenzierung (Hierarchie) anhand von Mitgliederzahl und Stellung der Kehilla (Traditionsträchtigkeit) weg. Manche sog. »Landgemeinden « avancierten regelrecht zu kulturellen und geistigen Zentren mit regionalem Einzugsbereich, wie Ichenhausen oder Heidingsfeld, die sich im Zeitalter der Aufklärung durch die Errichtung prachtvoller Synagogenbauten selbstbewusst nach außen hin präsentierten. ${ }^{110}$

Als etwa Heidingsfeld 1780 seine neue Synagoge einweihen konnte, war damit der Höhepunkt einer jahrhundertelangen, weitgehend kontinuierlichen Besiedlungsgeschichte und kulturellen Entwicklung erreicht. Seit Mitte des 15. Jahrhunderts eine unabhängige Gemeinde, hatte Heidingsfeld die 1561 aus dem nahegelegenen Würzburg vertriebenen Juden aufgenommen und wurde, da zahlenmäßig neben Fürth die bedeutendste Gemeinde in Franken, im 17. Jahrhundert Sitz eines Oberrabbinats. Die Gemeinde verfügte somit nicht nur über ein institutionalisiertes Gemeindeleben, sondern über weitreichenden Einfluss und einen eigenen Ritus. 1805 erreichte die Gemeinde mit rund 600 Personen ihren Höchststand, die mehr als 20 Prozent der Gesamtbevölkerung des Städtchens ausmachten. ${ }^{111}$

Wirtschaftliches Zentrum hingegen blieb weiterhin der Absatzmarkt Würzburg, den die jüdischen Händler mit Landesprodukten u. a. versorgten. Da ihnen die Niederlassung innerhalb der Stadtgrenze verwehrt war, legten sie den Weg in die Stadt vielfach täglich zurück. ${ }^{112}$

Die für die voremanzipatorische Epoche jüdischer Geschichtsschreibung durchaus kennzeichnende Divergenz von Zentrum/Peripherie in kulturell-geistiger einerseits und wirtschaftlich-administrativer Hinsicht andererseits

110 Annette Weber: Altfromm und/oder aufgeklärt? Zur Entwicklung von Landsynagogen und Gemeindekultur in der Zeit der Aufklärung und Emanzipation. In: Judentum und Aufklärung in Franken. Hg. vom Bezirk Mittelfranken durch Andrea M. Kluxen, Julia Krieger und Daniel Goltz. Würzburg: Ergon Verlag 2011, S. 131-155, hier: S. 135.

111 Pinkas Hakehillot, Germany, Vol. 1 (wie Anm. 109), S. 461-464.

112 Leser Kraft aus Heidingsfeld hat in seiner, unmittelbar eigene Erlebnisse verarbeitenden Wiedergabe der Würzburger Hepp-hepp-Ausschreitungen (1819) die Ankunft der aus Heidingsfeld, Höchberg etc. stammenden »Landjuden« (לאנדעס יהודים) für den 13. Av nachvollzogen, als sich der in den Gassen zusammenrottende Mob zu deren Verfolgung anschickte: M[oses] L[oeb] Bamberger: Beiträge zur Geschichte der Juden in Würzburg-Heidingsfeld. Würzburg: Frank 1905, S. 19. 
schwindet im Laufe des 19. Jahrhunderts. Als Würzburg 1808 jüdischen Zuzug bedingt, nämlich unter Nachweis eines beträchtlichen Vermögens, gestattet und 1816 Sitz des vormals in Heidingsfeld installierten Rabbinats wird, etabliert sich die Hauptstadt des Untermainkreises bzw. Unterfrankens auch aus jüdischer Sicht in jeder Hinsicht als Zentrum. Die Heidingsfelder Gemeinde mit ihrer rückläufigen Einwohnerzahl sinkt zur Peripherie herab, das Bewusstsein einstiger Größe und Bedeutsamkeit manifestiert sich forthin in einem bewusst jegliche Neuerung ablehnenden Traditionsdünkel. ${ }^{113}$

Im jüdischen Sprachgebrauch kontrastierte laut Tendlaus Sprichwörter und Redensarten deutsch-jüdischer Vorzeit der »Landsmann« mit dem »Killemann«, der dort mit »städtischem Juden« wiedergegeben wird, allerdings mit der Anmerkung, dass zur Gründung einer »Kehilla« (Gemeinde) lediglich ein Quorum von zehn Männern (Minjan) nötig sei. ${ }^{114}$ Tatsächlich bezeichnete der Begriff »Killemann« das Mitglied einer funktionstüchtigen, etablierten Gemeinde mit weitgehend institutionalisiertem Leben, der über ausreichend Kenntnis bzw. Zugang zu einer rabbinischen Autorität verfügte, um die religiösen Vorschriften zu befolgen. Dass es sich dabei keineswegs um den Bewohner eines von der staatlichen Obrigkeit anerkannten Hauptortes in politischer, ökonomischer und kultureller Hinsicht oder gar einen Großstädter handeln musste, zumal in der von Tendlau als »Vorzeit« apostrophierten vor- bzw. frühemanzipatorischen Epoche, dürfte aus den vorangegangenen Ausführungen klar hervorgegangen sein. Unter »Landsmann«, von Tendlau mit »Dorfjude« eingedeutscht, verstand man folglich den Bewohner einer (agrarisch geprägten) Ortschaft ohne bestehende jüdische Gemeinde bzw. nennenswerte Gemeindestruktur oder rabbinische Autorität. Der »Landsmann« galt seinen urbanen Glaubensbrüdern, wohl nach dem Vorbild des »am ha'aretz« konzipiert, als unbedarft, »besonders in Rücksicht auf religiöses Leben «, ${ }^{115}$ aber auch seinen alltäglichen Gewohnheiten, wie Hygienestandard und Umgangsformen, als unkultiviert. ${ }^{116}$ In Ansprüchen und Lebensweise dem Bauern gleichgestellt, befand sich der »Landsmann « am unteren Ende der gesellschaftlichen Skala. Die Bezeichnung »Landsmann« war keineswegs wertneutral, sondern abschätzig.

113 S. dazu etwa Der Israelit Jg 3, Nr 36 (3. Sept. 1862), S. 285 (Weber, Altfromm und/oder aufgeklärt? [wie Anm. 110], S. 136: Fn. 11).

114 Abraham Moses Tendlau: Sprichwörter und Redensarten deutsch-jüdischer Vorzeit. Als Beitrag zur Volks-, Sprach- und Sprichwörter-Kunde. Aufgezeichnet aus dem Munde des Volkes und nach Wort und Sinn erläutert. Frankfurt a.M.: H. Keller 1860, S. $205 f .:$ Nr 652.

115 Ebd., S. 206: Nr 652 (zur Redensart: »Er macht dem Landsmann sein Nachbrōche!«)

116 »Wenn der Bauer kaan Kerb (Kerwe, Kirwe, Kirchweihe) hätt, und der Landsmann [...] kaan Pesach, kämen sie aus dem Dreck nit heraus« (ebd., S. 113f.: Nr 370). 
In welche Zeit die einschlägigen Redensarten zurückreichen, ob erst die Annahme bürgerlicher Umgangsformen und ein höherer Lebensstandard die einfache, oft karge Lebensweise der Landbevölkerung als unzureichend erscheinen ließ oder bereits Jahrzehnte zuvor der »Killemann« seiner Empörung über die mangelnde religiöse Observanz der ungebildeten jüdischen Schicht darin Ausdruck verlieh, lässt sich nicht genau ermitteln; aus Tendlaus Erläuterungen spricht ganz deutlich eine aufklärerisch-bürgerliche Gesinnung, wenn auch der Tadel an der religiösen Lebensführung, wie wir wissen, viel älter war.

Bereits in hebräischen Quellen aus dem Mittelalter wird sorgfältig zwischen »kehilla« und »jischuw « unterschieden, wobei letzterer eine Ortschaft mit geringer Einwohnerzahl bezeichnet, insbesondere aber einen Ort, an dem die Zahl der jüdischen Einwohner nicht zur Bildung eines Minjan ausreicht. In religiösen Angelegenheiten waren die Bewohner eines Jischuw auf die nächste Kehilla angewiesen, die sich jedoch nicht immer in unmittelbarer Nähe befand. Eine nicht geringe Herausforderung für den Aufrechterhalt jüdischen Lebens stellten die von den Zentren abgeschieden wohnenden Juden dar, fand sich doch nicht vor Ort, was dazu nötig war: koschere Zeugen, Fest- und Feiertagsgebete, Mikwaot, etc. Kein Wunder also, dass die religiösen Sitten in den kleinen Ortschaften nicht immer dem rabbinischen Standard entsprachen. Die Juden auf den Dörfern und in den Jischuwim wüssten nicht zu beten, warnt etwa der Talmudist und Rechtsentscheider Jakob haLevi Moelin (MaHaRIL, 1375-1427) - und nennt sie in einem Atemzug mit den Gesetzesunkundigen (»am ha'aretz«). ${ }^{117}$ Dem unbeschlagenen jüdischen Dörfler - nicht zu verwechseln mit dem (christlichen) Bauern, dem »kafri« - begegnet man später, selten aber doch, in osteuropäisch-jüdischen Parabel- und Anekdotensammlungen, etwa den Gleichniserzählungen des Maggid von Dubno, Jakob Kranz (1741-1804), oder in Chanoch Henoch haKohen Levins (1798-1870) Chashava le-tova. ${ }^{118}$ Eine eingehende Prüfung jiddischer Quellen aus

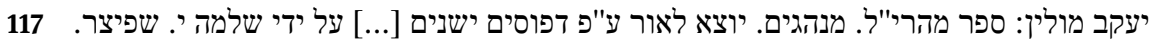

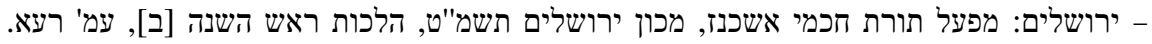
Weitere Belegstellen zur Verwendung des Begriffs »ischuw« finden sich in Eliezer Ben Yehuda: A Complete Dictionary of Ancient and Modern Hebrew. Thesaurus totius hebraitatis et veteris et recentioris. Jerusalem 1948-1959, Bd 4, S. 2181.

יעקב דובנא: ספר כוכב מיעקב. באורים ופרושים, פשטים ודרושים על סדר ההפטרות [...]. 118

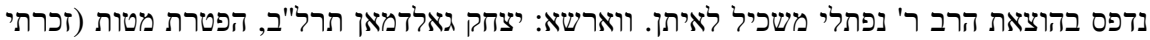

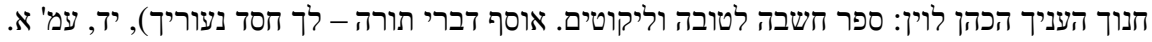

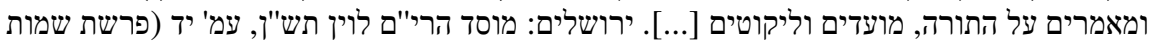

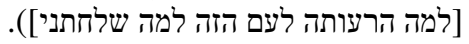


voremanzipatorischer Zeit steht noch aus und kann im Rahmen dieser Arbeit nicht geleistet werden. ${ }^{119}$

Der »frühneuzeitliche Verfeinerungsprozess der Umgangsformen «, ${ }^{120}$ in dessen Folge »die Juden« in den Augen der tonangebenden christlichen Schichten und Mehrheitsbevölkerung als »unsauber « im weiten Sinn ${ }^{121}$ erschienen, hatte auch die jüdische Bevölkerung erreicht und zu einer inneren Differenzierung nicht nur anhand des Kriteriums religiöser Observanz geführt.

In seinem Stück Der Spiegel für Israeliten (1817), einer Entgegnung zu Sessas judenfeindlicher Posse Unser Verkehr, lässt der fränkische Aufklärer Simon Höchheimer die Hausiererin Sorl und deren Tochter Bele auftreten. ${ }^{122}$ Letztere wird krätzig und in Lumpen gekleidet von ihrer Mutter im Handel mit Kurzwaren unterwiesen, zu dessen Ausübung auch die Bitte um Almosen und abgelegte Kleider im Gegenzug für gute Wünsche gehört. Dass die Familie kaum besser als Bettler lebt, scheint sie nicht zu bekümmern. Zuhause, wo man auf altem und schmutzigem Stroh schläft und sich ein einfaches Mahl zubereitet, halte man sich ohnehin nur wenig auf. Recha (!), die gebildete Tochter des erfolgreichen Fabrikanten Nathan (!) Weismann, fühlt sich von der schamlosen Zudringlichkeit und den Vertraulichkeiten der Hausiererin peinlich berührt und empört sich über deren Missachtung des Standesunterschieds. Aus dem Dialog wird deutlich, dass weniger die materiellen Bedingungen als überkommene Ansichten und Gewohnheiten einer - aus Rechas bürgerlicher Sicht - »Verbesserung « der Lebensumstände im Wege stehen. Eine gewisse Sorgfalt in Kleidung und Körperhygiene besitzt ebenso wie eine Ausbildung keinen Wert. Den Vorschlag, die Tochter in Dienst zu geben, lehnt die Mutter entschieden ab; eine Anstellung schädige das

119 Zum »Dorfjuden« in der jüdischen Folklore s. Haim Schwarzbaum: Studies in Jewish and World Folklore. Berlin: De Gruyter 1968, S. 23ff.; ders.: The Village Jew in Jewish Folkways and Folklore. In: Mahnayim 53 (1961), S. 116-122 (hebr.). - Schwarzbaums Zugang ist maßgeblich ahistorisch und typologisch; philologische Gesichtspunkte bleiben ebenso unberücksichtigt wie regionale Unterschiede.

120 Diemling, »Daß man unter so viel tausend Menschen so fort einen Juden erkennen kann« (wie Anm. 81), S. 83.

121 "Sauberkeit in diesem Sinne bedeutete, ganz wie Schudt das beschreibt, eine ordentliche Haltung in der Mehrdeutigkeit dieses Wortes, die Körperhaltung, Gesten, Sprache und das Gewand umfaßte. Das Tragen von schlampig gelegter Kleidung und vom Straßendreck kotiger Schuhe, das er an den Juden beanstandet, zeigte einen Mangel an Anstand, verfeinerten Sitten und Selbstdisziplin, der Schudt und seine christlichen Zeitgenossen abstieß und sie Juden als unsauber wahrnehmen ließ« (ebd., S. 84).

122 Simon Höchheimer: Der Spiegel für Israeliten. Ein Gegenstück zu der Posse »Unser Verkehr«. Nürnberg, Berlin: Campe 1817, S. 38-43. 
Ansehen der Familie und somit die Chancen der Tochter auf eine baldige Heirat. Während Sorles Achtung für Selbstbestimmung und Unabhängigkeit aus traditionell-jüdischen Vorstellungen von Ehrbarkeit herrührt, hält Recha den Widerstand der Hausiererin für falschen Dünkel.

Sorl und ihr Mann, der Hausierer Jaukuf, sind als Repräsentanten der sog. "pharisäischen « Klasse ${ }^{123}$ von Juden konstruiert, die »nur in Religion und Erwerb leben« und »die Religion, ihre Zeremonien, ihre Gebräuche, und die talmudischen Lehren über alles schätzen. "Selbst nicht gebildet, suchen sie »Heil, Trost, Glück und Seligkeit « in einer Religiosität, in die sich »Mißbräuche, Schwärmerei, Aberglaube u.s.w. eingeschlichen « haben. ${ }^{124}$ Durch »Bethen, Fasten u. dgl., durchs Lästern, durch Schmäh- und Strofreden [!] an den Nichtfrommen « ${ }^{125}$ suchen sie sich ihren Teil an der kommenden Welt zu sichern. Auf diese religiöse Weltsicht reagiert Recha empört, von der wiederholt thematisierten Nachlässigkeit in äußeren Dingen, dem Schmutz und der Krätze, zeigt sie sich, zumal offen dargeboten, angewidert. Eine »Gleichstellung, die sich ungebildete Juden gegen Juden erlauben« weist sie als »unerträglich « ${ }^{126}$ zurück; ihre »jüdische[.] Religion ${ }^{127}$ sei kein wahrer Grund, sich Sorl und Bele zugehörig zu fühlen.

Fest steht, dass die »Landjuden« sowohl aus Perspektive der im Verband funktionstüchtiger, teils traditionsträchtiger Gemeinden lebenden Schutzjuden als auch in den Augen der staatlichen Obrigkeit und der christlichen Mehrheitsgesellschaft zusehends zum sozialen Phänomen gerieten, oder besser gesagt: zum Sozialfall. Wohlmeinende wie feindselige Stimmen nahmen gleichermaßen die Armut einer ländlichen Bevölkerung wahr, die gerade noch am Rande der anerkannten jüdischen Gemeinschaft (Schutzbrief) stand oder schon - qua wirtschaftlichem Unvermögen - davon ausgeschlossen war.

Die Suche nach »den Landjuden « in älteren Quellen ist deswegen nicht ergiebig, weil Judenfiguren an sich kaum individuelle Züge besaßen, auf ihre Vertreter-

123 Höchheimer lässt den aufgeklärten Buchhalter Jonas die zeitgenössische Judenschaft in vier Klassen einteilen, die Nathan Weismann als die »pharisäische«, die »heterodoxe«, die »orthodoxe« und die »philosophische« bezeichnet (ebd., S. 60). - Eine - wenn auch abweichende - Einteilung in vier Klassen hatte 1812 bereits Sabattia Joseph Wolff vorgenommen und war unter den Anhängern der jüdischen Reform verbreitet (Michael A. Meyer: Jüdische Gemeinden im Übergang. In: Deutsch-jüdische Geschichte in der Neuzeit, Bd II [wie Anm. 12], S. 96-134, hier: S. 109f.).

124 Alle Zitate Höchheimer, Der Spiegel für Israeliten (wie Anm. 122), S. 54.

125 Ebd., S. 41.

126 Ebd., S. 43.

127 Ebd., S. 42. 
funktion beschränkt blieben und als Träger unterschiedlicher Bedeutungszuschreibungen agierten, die traditionell vermittelt und antijüdisch konnotiert waren: Juden standen für »Reichtum «, »Verarmung der christlichen Bevölkerung «, »Wucher«, »Geldgier«, »Aberglaube«. ${ }^{128}$ Neben ihrer religiösen »Abgeschiedenheit« stach ihre wirtschaftliche Sonderexistenz, die Beschränkung auf Handel und Geldverleih, negativ hervor. In manchen Regionen waren die Begriffe »Jude» und »Hausierer « austauschbar. ${ }^{129}$

Erst aus der inhärent städtisch-bürgerlichen Perspektive der literaturschaffenden gebildeten Schicht formt sich der Komplex »Land « als kulturell inferiores Gegenstück oder verklärte Idylle einer überschaubaren Welt, als dessen Spezialfall sich die Literarisierung eines "Landjudentums« darstellt.

Obwohl diese Ansichten als grobe Richtlinien ihre Berechtigung besitzen, lohnt sich der Blick zurück, die Rückschau auf eine Zeit, in der es mehr Landjuden als »das Landjudentum« gab.

128 Gutsche, Zwischen Abgrenzung und Annäherung (wie Anm. 72), S. 387. - Gutsche hält für die Literatur des 17. Jahrhunderts fest: "Juden erscheinen in den [...] untersuchten Texten stets als das Andere, an das man sich zwar annähern kann, eine Aufgabe der Differenzierungszuschreibungen findet jedoch nicht statt. [...] Die Juden erscheinen aufgrund ihrer religiösen Andersartigkeit nicht nur als fremd, sondern vor dem Hintergrund des nie in Frage gestellten Suprematieanspruches der christlichen Kirche als inferior « (ebd.).

129 S. o.: »Es kamen ein paar Land-Juden als sogenannte Haußirer mit unterschiedlichen Sachen zu meinem Herrn [...]« (Die redende Thiere. Drittes Gespräch [wie Anm. 76], S. 120). 